\title{
Feel the burn-muscle metabolite couples exercise to heat production
}

The discovery of a small molecule secreted by skeletal muscle cells could help explain in part how physical activity is effective for prevention and treatment of conditions such as obesity and diabetes mellitus. From their analysis of muscle metabolites, an international research consortium found that exercise triggers the production of $\beta$-aminoisobutyric acid (BAIBA), which in turn promotes heat production in white adipose tissue and breakdown of lipids by the liver through a common transcriptional mechanism.

Previous work on the benefits of exercise indicated that the transcriptional coactivator PGC-1a regulates musclespecific expression of genes involved in metabolism. Nonetheless, the mechanism whereby the molecular effects occurring in muscle during exercise are conveyed to other tissues remained poorly understood. Roberts and colleagues, therefore, took a metabolomic approach to search for PGC-1 $\alpha$-dependent signalling molecules.

The researchers screened the culture media of muscle cells overexpressing
PGC- $1 \alpha$ and identified the valine metabolite BAIBA as a candidate molecule. Using a combination of cell culture and mouse models, they showed that BAIBA induced the expression of brown-adipose-specific genes in white adipose tissue (so-called 'browning'), as well as genes required for hepatic $\beta$-oxidation of fatty acids. The effects of BAIBA were dependent on the transcription factor PPARa and could be reproduced in mice undergoing prolonged periods of exercise.

Notably, these laboratory findings were confirmed in humans. The investigators demonstrated that plasma concentrations of BAIBA were inversely correlated with several measures of metabolic risk (for example, levels of glucose, insulin resistance and lipid profiles) in a group of 2,067 individuals. In addition, they conducted metabolic profiling before and after a 20 -week exercise programme among 80 sedentary people and found a $17 \%$ increase in circulating levels of BAIBA following the intervention.

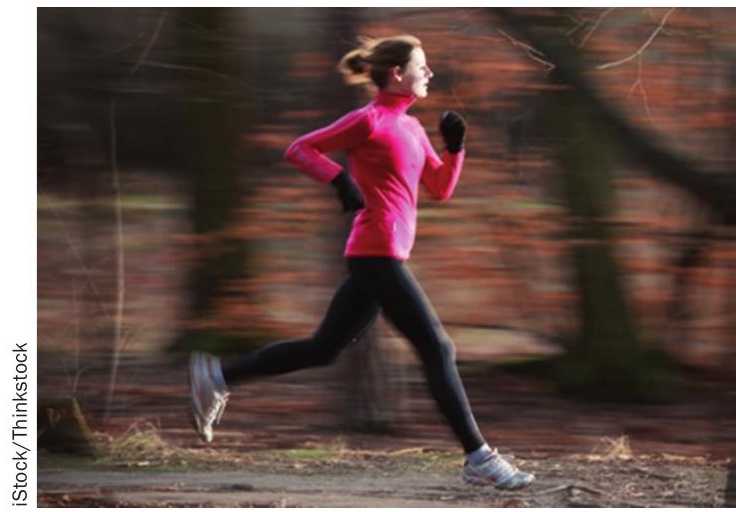

Roberts and co-workers conclude that conversion of valine to BAIBA by skeletal muscle could contribute to the known metabolic benefits of exercise and suggest that this pathway represents a novel target for therapeutic intervention.

Vicky Heath

Original article Roberts, L. D. et al. $\beta$-aminoisobutyric acid induces browning of white fat and hepatic $\beta$-oxidation and is inversely correlated with cardiometabolic risk factors. Cell Metab. 19, 96-108 (2013) 